\title{
Duration of diabetes and cardiorenal efficacy of liraglutide and semaglutide: A post hoc analysis of the LEADER and SUSTAIN 6 clinical trials
}

\author{
Subodh Verma $\mathrm{MD}^{1}$ (1) | Stephen C. Bain $\mathrm{MD}^{2}$ (ㅇ | Tea Monk Fries $\mathrm{MD}^{3}$ | \\ C. David Mazer MD ${ }^{1}$ | Michael A. Nauck MD ${ }^{4}$ — | Richard E. Pratley MD ${ }^{5}$ ( ) \\ Søren Rasmussen $\mathrm{PhD}^{3} \mid$ Hans A. Saevereid MD ${ }^{3}$ | Bernard Zinman $\mathrm{MD}^{6}$ ๑ | John B. Buse MD
}

${ }^{1}$ Department of Anesthesia, Division of Cardiac Surgery, St. Michael's Hospital and University of Toronto, Toronto, Ontario, Canada

${ }^{2}$ Institute of Life Science, Swansea University Medical School, Swansea, UK

${ }^{3}$ Novo Nordisk A/S, Søborg, Denmark

${ }^{4}$ Diabetes Center Bochum-Hattingen, St Josef Hospital (Ruhr-Universität Bochum), Bochum, Germany

${ }^{5}$ Florida Hospital Translational Research Institute for Metabolism and Diabetes, Orlando, Florida

${ }^{6}$ Lunenfeld-Tanenbaum Research Institute, Mt. Sinai Hospital, University of Toronto, Toronto, Ontario, Canada

${ }^{7}$ Department of Medicine, University of North Carolina School of Medicine, Chapel Hill, North Carolina

\section{Correspondence}

Subodh Verma MD, PhD, FRCSC, University of Toronto, St. Michael's Hospital, 30 Bond St,

8th Floor, Bond Wing, Toronto, Ontario, M5B 1W8, Canada.

Email: vermasu@smh.ca

Funding information

The LEADER and SUSTAIN 6 trials were funded by Novo Nordisk.
Cardiovascular risk reduction with liraglutide and semaglutide in patients with type 2 diabetes was demonstrated in the LEADER (ClinicalTrials.gov: NCT01179048) and SUSTAIN 6 (ClinicalTrials. gov: NCT01720446) cardiovascular outcome trials. This post hoc analysis assessed the impact of diabetes duration ( $<5,5$ to $<15,15$ to $<25$ and $\geq 25$ years at baseline) on cardiorenal efficacy of these human glucagon-like peptide- 1 analogues using a Cox proportional hazards model. Proportions of patients in the LEADER trial across diabetes duration strata were $15 \%(<5$ years, $\mathrm{n}=1377$ ), $50 \%$ ( 5 to $<15$ years, $\mathrm{n}=4692), 27 \%$ (15 to $<25$ years, $\mathrm{n}=2504$ ) and $8 \%$ ( $\geq 25$ years, $n=748)$; corresponding proportions in the SUSTAIN-6 trial were $13 \%(<5$ years, $n=422), 48 \%$ ( 5 to $<15$ years, $n=1582$ ), $30 \%$ ( 15 to $<25$ years, $n=977$ ) and $10 \%$ ( $\geq 25$ years, $n=316$ ). Overall, longer diabetes duration was associated with higher age; higher prevalence of females; history of ischaemic stroke, peripheral arterial disease and insulin use; and inferior renal function. There was an increased frequency of major adverse cardiovascular events (MACE), expanded MACE and nephropathy events with increasing diabetes duration. Liraglutide and semaglutide consistently reduced the risk of cardiorenal outcomes across categories of diabetes duration ( $P$-interaction was not significant for all endpoints analysed).

\section{KEYWORDS}

cardiorenal, cardiovascular, diabetes duration, efficacy, LEADER, liraglutide, renal, semaglutide, SUSTAIN 6

\section{1 | INTRODUCTION}

The duration of type 2 diabetes is an important determinant of cardiovascular and renal outcomes. ${ }^{1-5}$ However, less is known about whether antihyperglycaemic agents exert a consistent benefit on these outcomes as duration of type 2 diabetes increases. LEADER $(N=9340)$ and SUSTAIN $6(N=3297)$ were large cardiovascular outcome trials in patients with type 2 diabetes that demonstrated the beneficial cardiovascular effects of liraglutide and semaglutide, respectively, vs placebo, in addition to standard of care treatment. In this post hoc analysis, we evaluated the impact of diabetes duration on key cardiovascular and renal outcomes following treatment with these two glucagon-like peptide-1 (GLP-1) analogues.

\section{2 | METHODS}


composite of cardiovascular death, nonfatal myocardial infarction (MI) and nonfatal stroke (major adverse cardiovascular events [MACE]). The key secondary endpoint (expanded MACE) also included coronary revascularization and hospitalization for unstable angina or heart failure. Renal outcomes were adjudicated and assessed as part of a prespecified secondary microvascular endpoint which included nephropathy events, defined as the new onset of macroalbuminuria, or doubling of serum creatinine level and an estimated glomerular filtration rate (eGFR) of at most $45 \mathrm{~mL} / \mathrm{min} / 1.73 \mathrm{~m}^{2}$, or the need for continuous renal-replacement therapy, or death from renal disease.

We evaluated the efficacy of liraglutide and semaglutide for these endpoints by diabetes duration at baseline using the following subgroups: $<5$ years, 5 to $<15$ years, 15 to $<25$ years and $\geq 25$ years. Hazard ratios (HRs) were estimated using a Cox proportional hazards model with treatment and diabetes duration sub-group as factors, and the interaction between both factors. Furthermore, for the SUSTAIN-6 trial, the Cox proportional hazards model was stratified for factors used for randomization. ${ }^{6}$ An interaction $P$ value less than 0.05 was deemed significant. No adjustment for multiplicity was performed. Analyses were repeated with adjustment for baseline covariates: age, antihyperglycaemic medication, geographic region, history of MI or stroke, renal function (eGFR), sex and smoking status. Sensitivity analyses were also performed with four sub-groups based on quartiles of diabetes duration.

\section{RESULTS}

\section{1 | Baseline characteristics}

In the LEADER trial, $15 \%(n=1377), 50 \%(n=4692), 27 \%(n=2504)$ and $8 \%(n=748)$ of patients had baseline diabetes duration of $<5$, 5 to $<15,15$ to $<25$ and $\geq 25$ years, respectively; corresponding proportions in the SUSTAIN-6 trial were $13 \%(n=422), 48 \%(n=1582)$, $30 \%(n=977)$ and $10 \%(n=316)$ for sub-groups $<5$ years, 5 to $<15$ years, 15 to $<25$ years and $\geq 25$ years, respectively. Baseline characteristics of patients according to duration of diabetes are shown in Table $1 \mathrm{~A}$ and $\mathrm{B}$. Across the diabetes duration strata in the LEADER and SUSTAIN 6 trials, mean diabetes duration was 3 years in the $<5$ years sub-group, 10 years in the 5 to $<15$ years sub-group, 19 years in the 15 to $<25$ years sub-group and 31 years in the $>25$ years sub-group (Table 1). Mean glycated haemoglobin (HbA1c) at baseline was similar across the strata of diabetes duration in both trials (8.4\%-8.8\%), with the lowest values recorded in the $<5$ years sub-groups (Table 1). Increasing diabetes duration was associated with increasing age, higher prevalence of females, modestly higher systolic blood pressure and greater insulin use (Table 1). The prevalence of some macrovascular complications, including peripheral artery disease, ischaemic stroke, carotid artery stenosis and coronary artery bypass graft (CABG) surgery was higher with longer duration of diabetes; however, this was not observed for $\mathrm{MI}$, heart failure and percutaneous coronary intervention (Table 1). Renal function (eGFR) at baseline worsened with increasing diabetes duration and, correspondingly, the prevalence of moderate or severe renal disease, including end-stage renal disease, increased with longer duration of diabetes (Table 1).

\section{2 | Risk of cardiorenal events according to diabetes duration regardless of treatment}

In the LEADER trial, MACE occurred more frequently with increasing diabetes duration ( $<5$ years: 167 patients [12.1\%], 3.1 events $/ 100$ patient years of observation [PYO]; 5 to $<15$ years: 631 patients [13.4\%], 3.5 events/100 PYO; 15 to <25 years: 383 patients [15.3\%], 4.1 events/100 PYO; $\geq 25$ years: 116 patients [15.5\%], 4.1 events/100 PYO). Compared with patients with diabetes duration of $<5$ years, HRs for MACE ( $95 \%$ confidence interval [Cl]) were $1.13(0.95 ; 1.34), 1.31(1.09 ; 1.57)$ and $1.30(1.03 ; 1.65)$ in patients with diabetes duration of 5 to $<15$ years, 15 to $<25$ years and $\geq 25$ years, respectively. Similar results were obtained for the expanded MACE outcome (<5 years: 273 patients [19.8\%], 5.1 events/100 PYO; 5 to <15 years: 981 patients [20.9\%], 5.5 events/100 PYO; 15 to <25 years: 566 patients [22.6\%], 6.0 events/100 PYO; $\geq 25$ years: 184 patients [24.6\%], 6.5 events/100 PYO). Compared with patients with diabetes duration of $<5$ years, HRs for expanded MACE $(95 \% \mathrm{Cl})$ were 1.06 (0.93; 1.21), 1.17 (1.02; 1.36) and 1.27 (1.05; 1.53) in patients with diabetes duration of 5 to $<15$ years, 15 to $<25$ years and $\geq 25$ years, respectively. Incidence rates for cardiovascular death were 1.0, 1.3, 1.7 and 1.8 per 100 PYO, and were 1.0, 1.5, 2.2 and 2.6 per 100 PYO for nephropathy, according to increasing duration of diabetes.

In the SUSTAIN 6 trial, MACE occurred most frequently in the sub-group of patients with the longest duration of diabetes, but the pattern of increasing risk of MACE with diabetes duration observed in the LEADER trial was less apparent $(<5$ years: 35 patients [8.3\%], 4.0 events/100 PYO; 5 to <15 years: 109 patients [6.9\%], 3.3 events/100 PYO; 15 to <25 years: 77 patients [7.9\%], 3.8 events/100 PYO; $\geq 25$ years: 33 patients [10.4\%], 5.1 events/100 PYO). Compared with patients with diabetes duration of $<5$ years, HRs for MACE $(95 \% \mathrm{Cl})$ were $0.80(0.55 ; 1.20), 0.83(0.55 ; 1.27)$ and $1.03(0.63 ; 1.70)$ in patients with diabetes duration of 5 to $<15$ years, 15 to $<25$ years and $\geq 25$ years, respectively,. The frequency of expanded MACE did, however, increase with diabetes duration ( $<5$ years: 51 patients [12.1\%], 5.9 events/100 PYO; 5 to <15 years: 212 patients [13.4\%], 6.5 events/100 PYO; 15 to <25 years: 145 patients [14.8\%], 7.2 events/100 PYO; $\geq 25$ years: 55 patients [17.4\%], 8.6 events/100 PYO). Compared with patients with diabetes duration of $<5$ years, HRs $(95 \% \mathrm{Cl})$ for expanded MACE were 1.11 (0.82; 1.53), $1.15(0.83 ; 1.61)$ and $1.29(0.87 ; 1.91)$ in patients with diabetes duration of 5 to $<15$ years, 15 to $<25$ years and $\geq 25$ years. Incidence rates for cardiovascular death were 1.4, 1.2, 1.3 and 2.0 per 100 PYO, and were 2.2, 1.8, 3.1 and 3.6 per 100 PYO for nephropathy, according to increasing duration of diabetes. Results were similar in the LEADER and SUSTAIN 6 trials in analyses adjusted for baseline covariates and in analyses using sub-groups based on quartiles of diabetes duration (data not shown).

\section{3 | Cardiorenal efficacy of liraglutide and semaglutide according to diabetes duration}

Figure 1 describes cardiorenal events in patients treated with liraglutide and semaglutide in the LEADER and SUSTAIN 6 trials, 
TABLE 1 Baseline characteristics according to duration of diabetes in the (A) LEADER and (B) SUSTAIN 6 trials

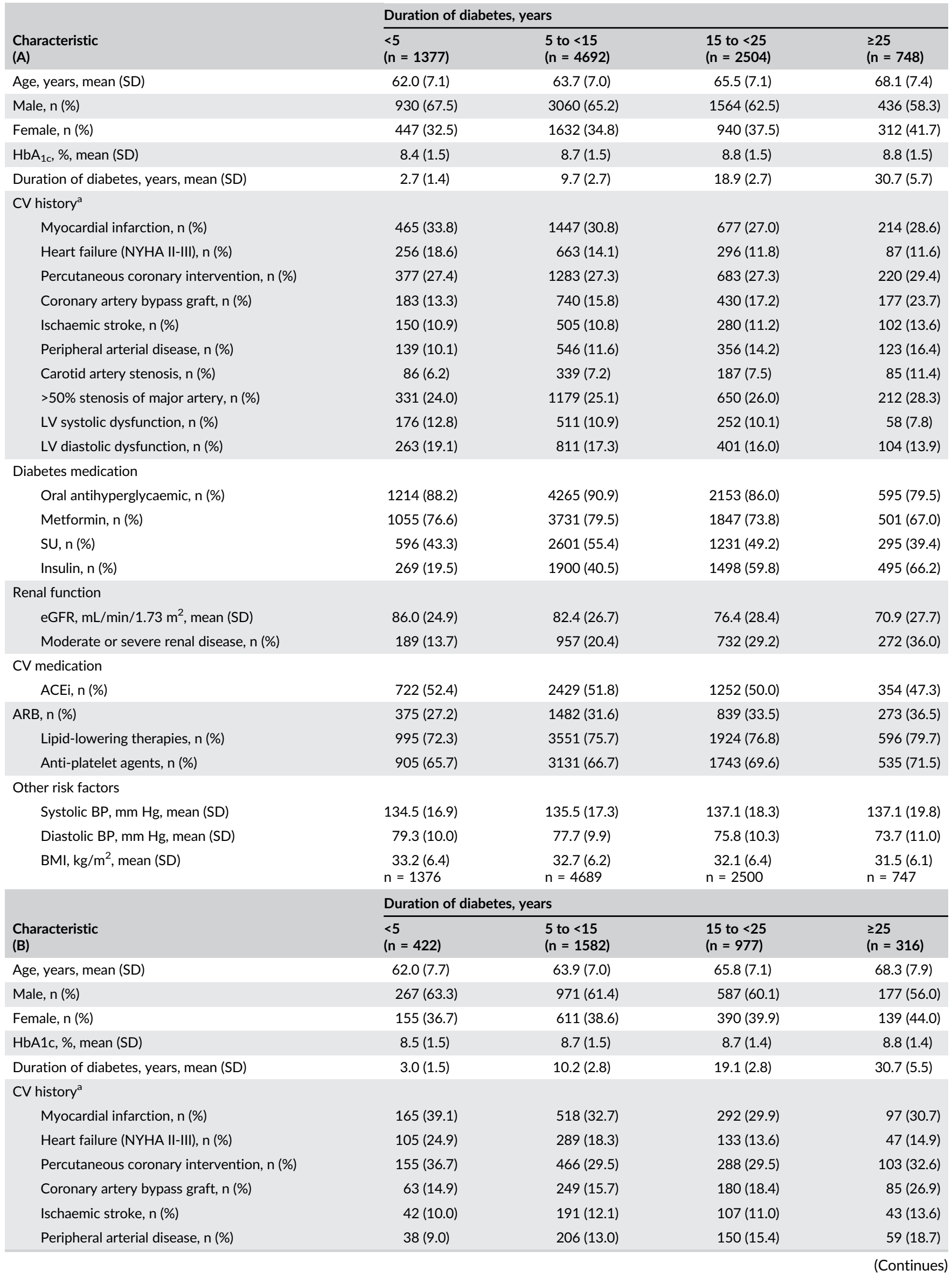


TABLE 1 (Continued)

\begin{tabular}{|c|c|c|c|c|}
\hline \multirow[b]{2}{*}{$\begin{array}{l}\text { Characteristic } \\
\text { (B) }\end{array}$} & \multicolumn{4}{|c|}{ Duration of diabetes, years } \\
\hline & $\begin{array}{l}<5 \\
(n=422)\end{array}$ & $\begin{array}{l}5 \text { to }<15 \\
(n=1582)\end{array}$ & $\begin{array}{l}15 \text { to }<25 \\
\text { (n = 977) }\end{array}$ & $\begin{array}{l}\geq 25 \\
(n=316)\end{array}$ \\
\hline Carotid artery stenosis, $\mathrm{n}(\%)$ & $35(8.3)$ & $129(8.2)$ & $95(9.7)$ & 35 (11.1) \\
\hline$>50 \%$ stenosis of major artery, $\mathrm{n}(\%)$ & $165(39.1)$ & $557(35.2)$ & $327(33.5)$ & $118(37.3)$ \\
\hline LV systolic dysfunction, n (\%) & $71(16.8)$ & $192(12.1)$ & $108(11.1)$ & $31(9.8)$ \\
\hline LV diastolic dysfunction, n (\%) & $108(25.6)$ & $342(21.6)$ & $170(17.4)$ & $50(15.8)$ \\
\hline Oral antihyperglycaemic, n (\%) & $355(84.1)$ & $1389(87.8)$ & $795(81.4)$ & $237(75.0)$ \\
\hline Metformin, $\mathrm{n}(\%)$ & $311(73.7)$ & $1228(77.6)$ & $688(70.4)$ & $190(60.1)$ \\
\hline SU, n (\%) & $180(42.7)$ & $737(46.6)$ & $386(39.5)$ & 107 (33.9) \\
\hline Insulin, $\mathrm{n}(\%)$ & $98(23.2)$ & $676(42.7)$ & $568(58.1)$ & $209(66.1)$ \\
\hline \multicolumn{5}{|l|}{ Renal function } \\
\hline $\mathrm{ACEi}, \mathrm{n}(\%)$ & $228(54.0)$ & $811(51.3)$ & $459(47.0)$ & $144(45.6)$ \\
\hline ARB, n (\%) & $104(24.6)$ & $522(33.0)$ & $358(36.6)$ & $127(40.2)$ \\
\hline Lipid-lowering therapies, n (\%) & $305(72.3)$ & $1196(75.6)$ & $760(77.8)$ & $260(82.3)$ \\
\hline Anti-platelet agents, n (\%) & $311(73.7)$ & $1158(73.2)$ & $695(71.1)$ & $245(77.5)$ \\
\hline \multicolumn{5}{|l|}{ Other risk factors } \\
\hline Systolic BP, mm Hg, mean (SD) & $133.3(15.9)$ & $135.4(17.1)$ & $136.4(17.3)$ & $137.5(18.4)$ \\
\hline Diastolic BP, mm Hg, mean (SD) & $79.01(9.8)$ & $77.7(9.9)$ & $76.1(10.1)$ & $74.1(9.8)$ \\
\hline $\mathrm{BMI}, \mathrm{kg} / \mathrm{m}^{2}$, mean $(\mathrm{SD})$ & $33.6(6.4)$ & $\begin{array}{c}33.2(6.0) \\
n=1578\end{array}$ & $\begin{array}{c}32.3(6.5) \\
n=974\end{array}$ & $31.6(5.9)$ \\
\hline
\end{tabular}

Abbreviations: ACEi, angiotensin-converting enzyme inhibitor; ARB, angiotensin receptor blocker; BMI, body mass index; BP, blood pressure; CV, cardiovascular; eGFR, estimated glomerular filtration rate; HbA1c, glycated haemoglobin; LV, left ventricular; NYHA, New York Heart Association; SD, standard deviation; SU, sulphonylurea.

Full analysis set, unless otherwise indicated. $P$ values were derived using chi-square test for differences between diabetes duration categories regardless of treatment group.

${ }^{a}$ Based on medical history as reported by the trial investigator for each patient.

Includes end-stage renal disease.

respectively, according to increasing duration of diabetes. Both GLP-1 analogues exhibited consistent benefits on the primary MACE endpoint, and on expanded MACE, cardiovascular death and nephropathy across diabetes duration strata, with no evidence of statistical heterogeneity $\left(P_{\text {interaction }}=\right.$ not significant for all endpoints) $($ Figure 1$)$. Similar results were obtained in analyses adjusted for baseline covariates (data not shown) and in analyses using sub-groups based on quartiles of diabetes duration (Figure S1).

\section{DISCUSSION}

The management of long-standing type 2 diabetes remains an important challenge. In addition to the difficulties of maintaining glycaemic control, long duration of diabetes is associated with an increase in cardiovascular complications and progressive renal decline, which in turn contribute to heightened rates of morbidity and mortality in these patients. ${ }^{1-5}$ While much is known about the association of diabetes duration with rates of complications, considerably less is known about the details of the efficacy and safety of antihyperglycaemic agents, such as GLP-1 analogues, as diabetes duration increases, particularly if
GLP-1 analogues have consistent cardiorenal efficacy across different durations of type 2 diabetes.

In an attempt to specifically address this subject, we conducted a post hoc analysis of two large cardiovascular outcome trials of liraglutide (LEADER) ${ }^{7}$ and semaglutide (SUSTAIN 6). ${ }^{6}$ Both trials demonstrated benefits of these GLP-1 analogues compared with placebo on the primary outcome of MACE (HR [95\% Cl]: 0.87 [0.78; 0.97] with liraglutide in LEADER and 0.74 [0.58; 0.95] with semaglutide in SUSTAIN 6). ${ }^{6,7}$ Furthermore, liraglutide was associated with a significant reduction in the risk of cardiovascular death (HR [95\% Cl]: 0.78 [0.66; $0.93]),{ }^{7}$ with consistent benefits observed in patients with atherosclerotic vascular disease, with or without a prior ischaemic event. ${ }^{8,9}$ Several mechanisms, including anti-inflammatory and anti-atherosclerotic effects, ${ }^{10-12}$ have been suggested to mediate the cardiovascular benefits of GLP-1 analogues that are observed in addition to those of conventional risk reduction therapies including lipid lowering. ${ }^{13}$

Our post hoc analyses of the LEADER and SUSTAIN 6 trials confirm that increasing duration of diabetes is associated with increased rates of cardiorenal outcomes. At baseline, increasing duration of diabetes was associated with a greater prevalence of peripheral artery disease, ischaemic stroke, history of CABG and renal disease. During 


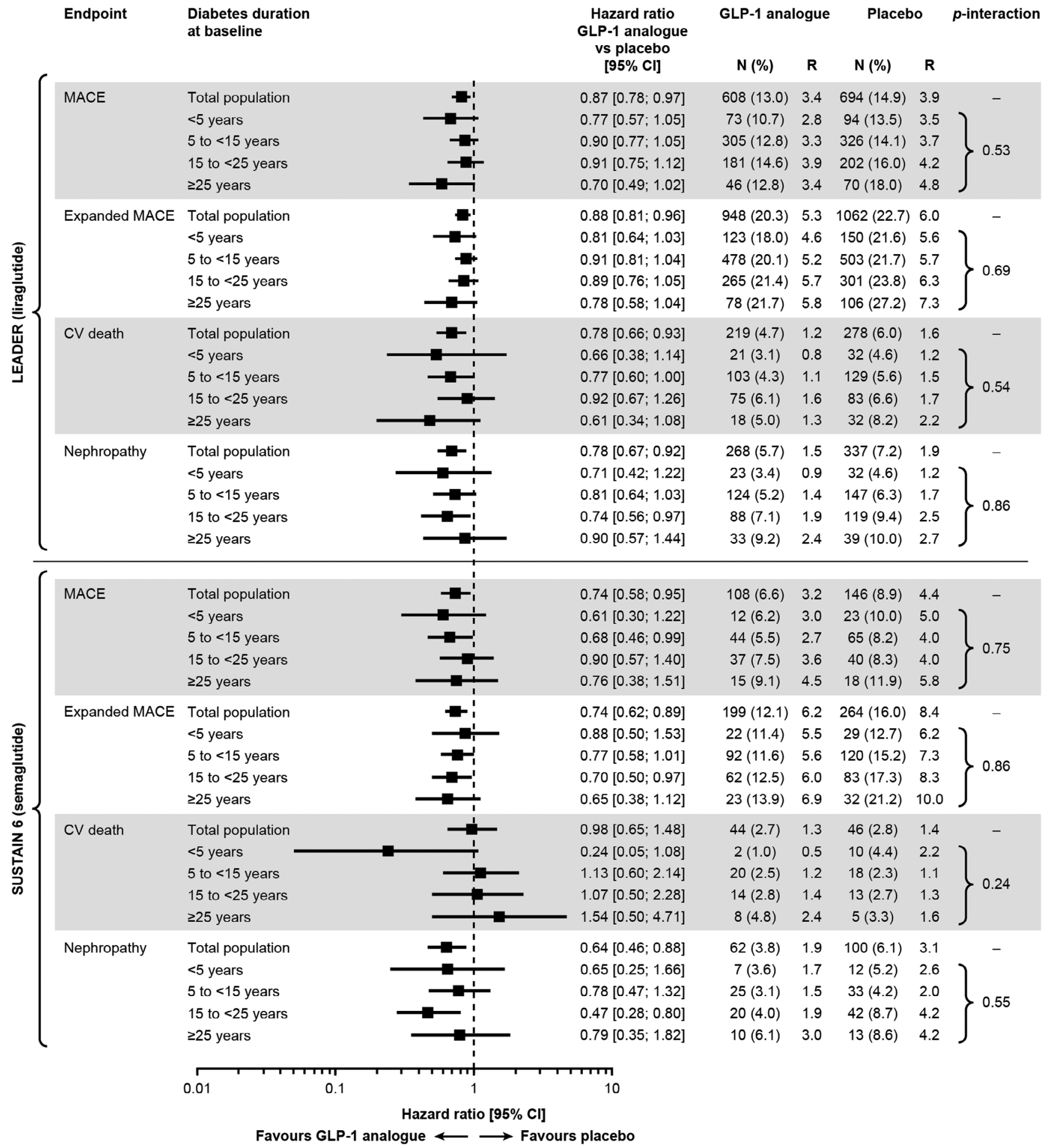

FIGURE 1 Risk of cardiorenal events in patients treated with liraglutide (upper panel) or semaglutide (lower panel) vs placebo, by diabetes duration at baseline. LEADER total population: 9340. LEADER total population with diabetes duration available: 9321 . SUSTAIN 6 total population (all patients with diabetes duration available): 3297. MACE comprised CV death, nonfatal MI and nonfatal stroke. Expanded MACE comprised MACE components plus coronary revascularization and hospitalization for unstable angina or heart failure. Nephropathy events were defined as new onset of macroalbuminuria or doubling of serum creatinine level and eGFR $\leq 45 \mathrm{~mL} / \mathrm{min} / 1.73 \mathrm{~m}^{2}$, the need for continuous renalreplacement therapy or death from renal disease. Abbreviations: $\mathrm{Cl}$, confidence interval; $\mathrm{CV}$, cardiovascular; eGFR, estimated glomerular filtration rate; GLP-1, glucagon-like peptide-1; MACE, major adverse cardiovascular events; N, number of patients with event; $R$, events per 100 patient years of observation

the trials, there was a trend for higher rates of MACE, expanded MACE, cardiovascular death and nephropathy with longer duration of type 2 diabetes. Importantly, both liraglutide and semaglutide exhibited consistent benefits on these cardiorenal endpoints irrespective of the duration of diabetes. For liraglutide, this finding of consistent benefits in apparently higher- and lower-risk patients is not concordant with the pre-specified sub-group analysis of the LEADER trial, which showed a significant interaction in favour of patients with 
established CVD.7 A more recent post hoc analysis of the LEADER trial showed that liraglutide reduced CV events both in patients with a history of $\mathrm{MI}$ or stroke and in those with established atherosclerotic cardiovascular disease without $\mathrm{MI}$ or stroke, indicating that the CV benefits of liraglutide are maintained across a clinically relevant continuum of risk in type 2 diabetes with atherosclerosis or prior ischaemic events. ${ }^{9}$ However, in the current analysis, patients with a shorter duration of diabetes had a higher prevalence of heart failure, myocardial infarction, and systolic or diastolic dysfunction at baseline, compared with those with a longer duration of diabetes, indicating previous events. Hence, it is difficult to make a direct comparison with the previously reported results.

The similar designs of the LEADER and SUSTAIN 6 trials facilitate comparison of our findings across the two trials, but this may also limit our findings to the patients enrolled in these trials. While not all glucagon-like peptide-1 receptor agonists (GLP-1RAs) have demonstrated cardiorenal efficacy, it could be of interest for future analyses to examine the impact of diabetes duration on the cardiorenal effects of GLP-1RAs across a wider range of trials and patients.

In summary, our analyses provide reassurance that the cardiorenal efficacy and safety of liraglutide and semaglutide are maintained over a broad range of patients, from those who are relatively newly diagnosed to those with long-standing type 2 diabetes.

\section{ACKNOWLEDGMENTS}

Editorial assistance was provided by Charlie Hunt and Izabel James, of Watermeadow Medical, an Ashfield Company, part of UDG Healthcare plc (supported financially by Novo Nordisk), during preparation of this article.

Results in this Brief Report have been published, in part, in abstract form, and were presented at the American College of Cardiology (ACC) Annual Scientific Session, March 16-18, 2019, New Orleans, Louisiana.

\section{CONFLICT OF INTEREST}

S. V. has received research grants and/or speaking honoraria from Boehringer Ingelheim/Eli Lilly, AstraZeneca, Janssen, Merck, Novartis, Novo Nordisk, Sanofi, Valeant and Amgen.

S. C. B. has received research grants as principal investigator, collaborator or consultant; has pending grants from Healthcare and Research Wales (Welsh Government) and Novo Nordisk; has received other research support from Healthcare and Research Wales (Welsh Government) and honoraria from Novo Nordisk, Sanofi, Lilly, Boehringer Ingelheim and Merck, and has an ownership interest in Gycosmedia (diabetes on-line news service).

H. A. S., T. M. F. and S. R. are full-time employees of Novo Nordisk A/S. T. M. F. and S. R. hold stock in Novo Nordisk A/S.

C. D. M. has received consulting fees from Amgen, Boehringer Ingelheim and OctaPharma.

M. A. N. has served on advisory boards; has consulted for AstraZeneca, Boehringer Ingelheim, Eli Lilly \& Co., Fractyl, GlaxoSmithKline, Servier, Menarini/Berlin Chemie, Merck, Sharp \& Dohme and Novo Nordisk; has served on speakers bureaus of
AstraZeneca, Boehringer Ingelheim, Eli Lilly \& Co., GlaxoSmithKline, Menarini/Berlin Chemie, Merck, Sharp \& Dohme, Novo Nordisk A/S and Sun Pharma; and his institution has received grant support from AstraZeneca, Boehringer Ingelheim, Eli Lilly \& Co., GlaxoSmithKline, Intarcia, Menarini/Berlin-Chemie, Merck, Sharp \& Dohme, Novartis Pharma and Novo Nordisk A/S.

R. E. P. has received research grants from Gilead Sciences, Lexicon Pharmaceuticals, Ligand Pharmaceuticals Inc., Lilly, Merck, Novo Nordisk, Sanofi-Aventis US LLC and Takeda; has acted as a speaker for AstraZeneca, Novo Nordisk and Takeda; and has acted as a consultant for AstraZeneca, Boehringer Ingelheim, Eisai, Inc., GlaxoSmithKline, Janssen Scientific Affairs LLC, Ligand Pharmaceuticals Inc., Lilly, Merck, Novo Nordisk, Pfizer and Takeda (all payments are made directly to his employer, Florida Hospital).

B. Z. has received consulting fees from Merck, Novo Nordisk, Sanofi-Aventis, Eli Lilly, AstraZeneca, Jansse, and Boehringer Ingelheim.

J. B. B. has been an advisor, with all fees paid to the University of North Carolina, to Adocia, AstraZeneca, Dance Biopharm, Eli Lilly, MannKind, NovaTarg, Novo Nordisk, Senseonics and vTv Therapeutics; has received grant support from Novo Nordisk, Sanofi and vTv Therapeutics; is a consultant to Neurimmune AG; is supported by a grant from the National Institutes of Health (UL1TR002489); and holds stock options in Mellitus Health, PhaseBio and Stability Health.

\section{Author contributions}

Statistical analyses were performed by Søren Rasmussen, and Subodh Verma prepared the first draft. All authors were responsible for the content and editorial decisions, were involved at all stages of manuscript development and approved the final version.

Patient level analysis data sets for the research presented in the publication are available from the corresponding author upon request.

\section{ORCID}

Subodh Verma (D) https://orcid.org/0000-0002-4018-8533 Stephen C. Bain (D) https://orcid.org/0000-0001-8519-4964 Michael A. Nauck (D) https://orcid.org/0000-0002-5749-6954 Richard E. Pratley (D) https://orcid.org/0000-0002-2912-1389 Bernard Zinman (D) https://orcid.org/0000-0002-0041-1876

\section{REFERENCES}

1. Fox CS, Sullivan L, D'Agostino RB Sr, Wilson PW, Framingham Heart Study. The significant effect of diabetes duration on coronary heart disease mortality: the Framingham Heart Study. Diabetes Care. 2004;27:704-708.

2. Herrington WG, Alegre-Diaz J, Wade R, et al. Effect of diabetes duration and glycaemic control on 14-year cause-specific mortality in Mexican adults: a blood-based prospective cohort study. Lancet Diabetes Endocrinol. 2018;6:455-463.

3. Kim JJ, Hwang BH, Choi IJ, et al. Impact of diabetes duration on the extent and severity of coronary atheroma burden and long-term clinical outcome in asymptomatic type 2 diabetic patients: evaluation by coronary CT angiography. Eur Heart J Cardiovasc Imaging. 2015;16: 1065-1073.

4. Wannamethee SG, Shaper AG, Whincup PH, Lennon L, Sattar N. Impact of diabetes on cardiovascular disease risk and all-cause 
mortality in older men: influence of age at onset, diabetes duration, and established and novel risk factors. Arch Intern Med. 2011;171: 404-410.

5. Zoungas S, Woodward M, Li Q, et al. Impact of age, age at diagnosis and duration of diabetes on the risk of macrovascular and microvascular complications and death in type 2 diabetes. Diabetologia. 2014;57: 2465-2474.

6. Marso SP, Bain SC, Consoli A, et al. Semaglutide and cardiovascular outcomes in patients with type 2 diabetes. N Engl J Med. 2016;375: 1834-1844.

7. Marso SP, Daniels GH, Brown-Frandsen K, et al. Liraglutide and cardiovascular outcomes in type 2 diabetes. N Engl J Med. 2016;375: 311-322.

8. Verma S, Bhatt DL, Bain SC, et al. Effect of liraglutide on cardiovascular events in patients with type 2 diabetes mellitus and polyvascular disease: results of the LEADER trial. Circulation. 2018;137:2179-2183.

9. Verma S, Poulter NR, Bhatt DL, et al. Effects of liraglutide on cardiovascular outcomes in patients with type 2 diabetes mellitus with or without history of myocardial infarction or stroke. Circulation. 2018; 138:2884-2894.

10. Drucker DJ. The cardiovascular biology of glucagon-like peptide-1. Cell Metab. 2016;24:15-30.

11. Rakipovski G, Rolin B, Nohr J, et al. The GLP-1 analogs liraglutide and semaglutide reduce atherosclerosis in $\operatorname{ApoE}(-/-)$ and $\operatorname{LDLr}(-/-)$ mice by a mechanism that includes inflammatory pathways. JACC Basic Transl Sci. 2018;3:844-857.

12. Nauck MA, Meier JJ, Cavender MA, Abd El Aziz M, Drucker DJ. Cardiovascular actions and clinical outcomes with glucagon-like peptide-1 receptor agonists and dipeptidyl peptidase-4 inhibitors. Circulation. 2017;136:849-870.

13. Verma S, Leiter LA, Mazer CD, et al. Liraglutide reduces cardiovascular events and mortality in type 2 diabetes mellitus independently of baseline low-density lipoprotein cholesterol levels and statin use: results from the LEADER trial. Circulation. 2018;138:1605-1607.

\section{SUPPORTING INFORMATION}

Additional supporting information may be found online in the Supporting Information section at the end of this article.

How to cite this article: Verma S, Bain SC, Monk Fries T, et al. Duration of diabetes and cardiorenal efficacy of liraglutide and semaglutide: A post hoc analysis of the LEADER and SUSTAIN 6 clinical trials. Diabetes Obes Metab. 2019;21:

1745-1751. https://doi.org/10.1111/dom.13698 